\title{
Stimulation of paralysed quadriceps muscles with sequentially and spatially distributed electrodes during dynamic knee extension
}

\author{
Marco Laubacher ${ }^{1,2^{*}}$ (D), Efe A. Aksoez ${ }^{1,2}$, Anne K. Brust ${ }^{3}$, Michael Baumberger ${ }^{3}$, Robert Riener ${ }^{2}$, \\ Stuart Binder-Macleod ${ }^{4}$ and Kenneth J. Hunt ${ }^{1}$
}

\begin{abstract}
Background: During functional electrical stimulation (FES) tasks with able-bodied (AB) participants, spatially distributed sequential stimulation (SDSS) has demonstrated substantial improvements in power output and fatigue properties compared to conventional single electrode stimulation (SES). The aim of this study was to compare the properties of SDSS and SES in participants with spinal cord injury (SCI) in a dynamic isokinetic knee extension task simulating knee movement during recumbent cycling.

Method: Using a case-series design, $m$. vastus lateralis and medialis of four participants with motor and sensory complete SCI (AIS A) were stimulated for 6 min on both legs with both electrode setups. With SES, target muscles were stimulated by a pair of electrodes. In SDSS, the distal electrodes were replaced by four small electrodes giving the same overall stimulation frequency and having the same total surface area. Torque was measured during knee extension by a dynamometer at an angular velocity of $110 \mathrm{deg} / \mathrm{s}$. Mean power of the left and right sides $\left(P_{\text {meanL,R }}\right)$ was calculated from all stimulated extensions for initial, final and all extensions. Fatigue is presented as an index value with respect to initial power from 1 to 0 , whereby 1 means no fatigue.

Results: SDSS showed higher $P$ mean $L, R$ values for all four participants for all extensions (increases of $132 \%$ in participant $\mathrm{P} 1,100 \%$ in $\mathrm{P} 2,36 \%$ in P3 and 18\% in P4 compared to SES) and for the initial phase (increases of 84\%, 59\%, $66 \%$, and $16 \%$, respectively). Fatigue resistance was better with SDSS for P1, P2 and P4 but worse for P3 ( 0.47 vs 0.35 , 0.63 vs $0.49,0.90$ vs 0.82 and 0.59 vs 0.77 , respectively).

Conclusion: Consistently higher $P_{\text {mean } L, R}$ was observed for all four participants for initial and overall contractions using SDSS. This supports findings from previous studies with AB participants. Fatigue properties were better in three of the four participants. The lower fatigue resistance with SDSS in one participant may be explained by a very low muscle activation level in this case. Further investigation in a larger cohort is warranted.
\end{abstract}

Keywords: Functional electrical stimulation, Spinal cord injury, Rehabilitation, Spatially distributed sequential stimulation, Knee dynamometer, Power output, Fatigue

*Correspondence: marco.laubacher@bfh.ch

${ }^{1}$ Institute for Rehabilitation and Performance Technology, Division of

Mechanical Engineering, Department of Engineering and Information

Technology, Bern University of Applied Sciences, Pestalozzistrasse 20, 3400

Burgdorf, Switzerland

${ }^{2}$ Sensory Motor Systems Lab, Department of Health Sciences and Technology,

ETH Zurich, 8000 Zurich, Switzerland

Full list of author information is available at the end of the article

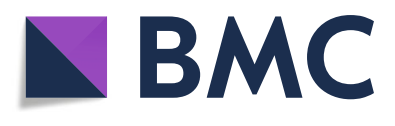

(c) The Author(s). 2019 Open Access This article is distributed under the terms of the Creative Commons Attribution 4.0 International License (http://creativecommons.org/licenses/by/4.0/), which permits unrestricted use, distribution, and reproduction in any medium, provided you give appropriate credit to the original author(s) and the source, provide a link to the Creative Commons license, and indicate if changes were made. The Creative Commons Public Domain Dedication waiver (http://creativecommons.org/publicdomain/zero/1.0/) applies to the data made available in this article, unless otherwise stated. 


\section{Background}

Mobilisation and exercise play an important role during the rehabilitation of persons after a spinal cord injury (SCI). On the one hand it is important to regain strength and mobility, and on the other hand it helps to prevent secondary complications [1-3]. Arm cycle ergometry or long wheelchair runs are often used for cardiovascular training and to maintain fitness [4], but these exercise modes only include upper body movement and the paralysed leg muscles remain inactive. As a result, blood pooling may occur in the lower limbs, and low blood pressure and deep venous thrombosis are possible consequences $[5,6]$. Functional electrical stimulation (FES) provides a means of mobilising intact lower leg structures and has been shown to be very effective in preventing these secondary complications when used regularly over a period of time [7-9]. Positive adaptations of bone mineral density and increased muscle strength are further benefits following electrical stimulation training $[10,11]$. Although these improvements help to reduce the risk of pressure sores and fractures, the application of FES is not yet fully integrated into the rehabilitation following severe spinal cord injury because the costs can be high and the health benefits may not be immediately apparent [12]. FES training is often replaced with other rehabilitation programmes or restricted to patients with incomplete lesions where motor learning and carry over effects are expected and observed [13].

Two major factors that limit the use and effectiveness of FES are the well-known problems of rapid muscle fatigue and low power output of artificial stimulation when used for functional tasks [14-18]. In the last 40 years, different approaches have been used to address these challenges. Pulse modulation, electrode placement or diversifications of electrodes are strategies applied to increase power or fatigue resistance. Most effort was placed on investigating the effects of pulse modulation, such as changing stimulation frequency, pulse width or amplitude. Constant, low frequency trains of pulses show better fatigue resistance compared to high frequency trains, but on the other hand the power output is significantly lower [19-22]. Adding doublets to stimulation trains increased the fatigue resistance and force production significantly, especially when applied to fatigued muscles [23-25]. Stochastic interpulse intervals showed inconsistent results. Depending on the specific task, fatigue resistance and power output were in some cases increased and in other cases worse compared to traditional stimulation patterns [26-29].

Stimulation intensity can also be controlled using pulse width or amplitude. High intensity is usually correlated to high power output $[15,30]$ but entails decreased fatigue resistance [31-33]. The increases in power, observed when stimulation intensity is increased, is related to a higher number of activated motor units, but the precise recruitment mechanisms are unclear. It is proposed that the enlarged cross sectional activation area with higher pulse widths results from increased electrical signal propagation within the muscles, while increased amplitude will increase the current density and the electrical field will reach deeper structures [31, 34, 35]. Since it has been observed that higher intensities can cause more antidromic impulses [36], investigations in modulating the stimulation amplitude often include changes in the pulse width to keep current and stimulation intensity constant.

Most investigations have shown some improvements in either power or fatigue but no study to date has demonstrated meaningful increases in both power and fatigue. Non-selective recruitment of motor units and poor intraand inter-muscular coordination leads to an exaggerated metabolic cost of electrically evoked contractions $[16,17]$. This problem also exists in able-bodied persons, where power drops $30 \%$ shortly after stimulation onset (compared to 50\% fatigue in SCI) [37]. This indicates that, in part, low power output and high fatigue result from the characteristics of artificial muscle stimulation. Surface electrodes are spatially fixed and the underlying motor units are activated synchronously as soon as the critical threshold is reached. Since the location of the electrodes is a crucial part of the application, even small changes in placement can change the power substantially [38-40].

The use of more electrodes and spatial differentiation, termed spatially distributed sequential stimulation (SDSS), has been shown to be very effective in power generation and fatigue reduction [41-43]. With distributed electrodes, the electrical field can be varied and more motor units will be activated, thus more power can be generated [44]. To date, several different strategies have been investigated. Placing the electrodes on synergistic muscles will produce more power by increasing the activated muscle mass $[9,42,43]$, while placing more electrodes on the same muscle belly will increase the number of activated fibres of the same muscle [44-47]. The synchronous activation of all motor units below the electrodes is one major issue in the poor fatigue resistance with FES. With distributed electrodes, it is possible to add a temporal shift between the pulses and decrease the stimulation frequency for each electrode, while maintaining the same overall stimulation frequency. This setup, with the alternated activation of more motor units, has been shown to increase fatigue resistance significantly in several studies and to maintain or even increase power output compared to conventional ES [44-47]. The lower stimulation frequency applied to each electrode allows for a lower ATP cost for each contraction and is more efficient in binding cross-bridges. It is believed that the lower frequency causes fewer problems in $\mathrm{Ca} 2+$ release than are observed with high frequency stimulation. Furthermore, the mechanism whereby the electric field is 
changed constantly might activate other neural circuits, which again activates some other muscle parts in the same muscle group $[44,48,49]$.

The increasing number of studies with positive outcomes regarding distributed electrode setups indicates that this strategy is a promising solution for the low power and the low fatigue resistance of conventional surface electrode stimulation. However, the number of studies with SCI participants is limited and the transfer of knowledge gained with able-bodied participants to $\mathrm{SCI}$ is another challenge [25]. Following an SCI there is a rapid loss of muscle mass and a change in muscle nerve physiology based on histological changes in muscle composition. Muscle atrophy is very specific in each muscle depending on fibre type, body composition and training status before injury. A decrease in muscle cross sectional area of $20-50 \% 6$ months after injury is usual for unloaded muscles and comparable to long bed rest or space flight in able-bodied persons. The histochemical changes take longer and become significant 6 to 12 months after injury. Thus, the proportional distribution of fast- and slowtwitch fibres does not change significantly during the first weeks after injury [50-52]. Clearly, these changes in physiological structure influence the interaction between electrode and muscle [53]. With non-isometric FES, the physical movement of muscle bulk is another factor affecting tissue resistance. This can induce the activation of other nerve fibres and reflexes, which can then disturb the functional movement. As a consequence, for power or fatigue related investigations with surface electrodes, it is very important to mimic the intended task as precisely as possible $[54,55]$.

The aim of this study is to compare the power output and fatigue properties of the quadricps femoris muscles in response to spatially distributed sequential stimulation (SDSS) versus traditional single electrode stimulation (SES) in four untrained participants with motor-complete spinal cord injury during a dynamic leg extension task simulating knee joint movement in recumbent cycling.

\section{Method}

Four participants with motor-complete spinal cord injury and an American Spinal Cord Injury Association (ASIA) impairment score (AIS) A were included (Table 1) according to a case series study design [56]. Each participant gave written informed consent. The study was approved by the local ethics committee (Ethics Committee of northwest/central Switzerland, Ref.-Nr: BASEC 2016-00394).

\section{Measurement instruments}

Measurements were conducted using an isokinetic dynamometer (Cybex IIa, Biodex Medical Systems Inc., USA) with an upgrade package (Humac Norm, Computer Sports Medicine Inc., USA). The dynamometer was set up for concentric quadriceps measurement. After seating, individual adjustments were made for ergonomic knee joint movement.

The dynamometer controlled the angular range of motion at the knee joint from 40 to $120 \mathrm{deg}$ (180 deg means straight leg) at an angular velocity of $110 \mathrm{deg} / \mathrm{s}$ at the knee joint, which is equivalent to a cycling cadence of $50 \mathrm{rpm}$. The dynamometer was interfaced to a PC running Matlab/Simulink and the Real-Time Toolbox (MathWorks Inc., USA), which recorded raw data of the knee angle, torque and time. These data were used to control the stimulation range and the stimulation parameters. A graphical user interface was implemented for setting up and controlling the stimulation device and the timing (Aksoz EA, Laubacher M, Binder-Macleod S, Hunt KJ, Design of an isokinetic knee dynamometer for evaluation of functional electrical stimulation strategies, submitted).

\section{Stimulation}

Before measurement, the skin was cleaned and the body hair shaved at the position of the electrodes. For each stimulated muscle, one motor point was detected prior to measurement with a stimulation pen (Motor Point Pen, Compex SA, Switzerland). Motor points and electrode positions were marked to ensure identical placement across the sessions. The investigated stimulation setup was similar to a previous study with able-bodied participants [44]. Two different electrode setups were compared: SES and SDSS. In both setups, participants were stimulated with rectangular bi-phasic pulses of constant $40 \mathrm{~mA}$ amplitude generated under PC control with an eight-channel stimulator (RehaStim, Hasomed $\mathrm{GmbH}$, Germany) with a pulse width range of $0-500 \mu \mathrm{s}$ ( $1 \mu$ s steps) and a frequency range of $0-100 \mathrm{~Hz}$. For the SES setup, a single pair of self-adhesive electrodes with a dimension of $9 \times 5 \mathrm{~cm}$ (Pals Platinum, Axelgaard Mfg. Co., LTD, USA) were placed on the motor points of the $\mathrm{m}$. vastus lateralis and medialis. Reference electrodes with the same size were placed $10-15 \mathrm{~cm}$ proximal of the corresponding muscle motor point and the frequency was set at $35 \mathrm{~Hz}$. In the SDSS setup, four small electrodes each with a size of $4.5 \times 2.5 \mathrm{~cm}$ were placed around the previously detected motor point. Each of the four electrodes was stimulated with a frequency of $8.75 \mathrm{~Hz}$ and a phase shift of $90 \mathrm{deg}$, which corresponds to the stimulation frequency of the SES setup of $35 \mathrm{~Hz}$ and using the same total electrode area. The SDSS stimulation order was always the same, i.e. from 1 to 4 , and the reference electrodes were the same as for the SES setup (Fig. 1). For both setups, stimulation was applied only during the kneeextension phase of the motion, over a knee-angle range of $55 \mathrm{deg}$ to $115 \mathrm{deg}$. In each session the pulse width was adapted to the participant according the familiarization procedure detailed below. For this study, the mean pulse 
Table 1 Characteristics of the four participants

\begin{tabular}{llllllll}
\hline Participant & Sex & Age $(\mathrm{yrs})$ & Height $(\mathrm{cm})$ & Body mass $(\mathrm{kg})$ & Time since injury (months) & Lesion level & Rehab-status \\
\hline P1 & $\mathrm{f}$ & 45.4 & 168 & 61.3 & 23.8 & T3 & Re-Reha \\
P2 & $\mathrm{m}$ & 22.9 & 187 & 80.0 & 5.3 & T6 & First-Reha \\
P3 & $\mathrm{m}$ & 48.4 & 178 & 75.0 & 5.8 & T2 & First-Reha \\
P4 & $\mathrm{m}$ & 27.5 & 183 & 65.0 & 10.6 & T5 & First-Reha \\
mean \pm sd & & $36.1 \pm 12.7$ & $179 \pm 8.2$ & $70.3 \pm 8.7$ & 10.9 & \\
\hline
\end{tabular}

First-Reha: Primary rehabilitation phase directly after injury;

Re-Reha: Second entry into the rehabilitation clinic, after being at home following primary rehabilitation

width applied was $213.4 \pm 41.2 \mu$ s for SES and $217.8 \pm$ $35.8 \mu$ s for SDSS.

\section{Procedure}

Measurements were conducted in two sessions with at least $24 \mathrm{~h}$ between each session. Within each session, each leg was stimulated with only one electrode configuration. Between the two independent leg measurements participants had a break of $15 \mathrm{~min}$. Stimulation and leg order (SES then SDSS vs. SDSS then SES; L then R vs. R then L) was chosen randomly. Motor point detection was conducted prior to the first measurement while participants were seated in their wheelchair. Participants where then transferred to the dynamometer system and individual adjustments to body proportions were made. Before each stimulation (leg and setup), a brief familiarization was conducted to determine stimulation parameters and to control the device setup.

The familiarisation started with a two-minute passive phase in which the measured leg was moved by the device without stimulation (non-stimulation phase, ns-phase). The data collected during this phase was used as a baseline measurement for the leg movement resistance. The next phase involved stimulation while the leg was being moved by the dynamometer. The pulse width was initially set at $45 \mu \mathrm{s}$ and was manually increased after every extension until the power output started to plateau. Stimulation was halted and the pulse width needed to produce the maximum power $\left(P W_{\max }\right)$ was noted. The pulse width used for the subsequent measurement was $80 \%$ of $P W_{\max }$ $\left(P W_{m 80}=0.8 * P W_{\text {max }}\right)$. After a 10 -min rest period following familiarisation, the actual measurement was commenced with an ns-phase of two min and a stimulation phase (st-phase) of six min. A second two min ns-phase completed the measurement.

\section{Outcomes and data analysis}

The measured torque together with the angular speed was used to calculate the gross power output $P_{m}$ generated during the stimulated knee extension. The power used to move the leg during the ns-phase was defined as $P_{n s}$ and the net power output of one stimulation cycle, $P_{\text {stim }}$, was then obtained as $P_{\text {stim }}=P_{m}-P_{n s}$. The mean power output over the stimulation angle range during one extension
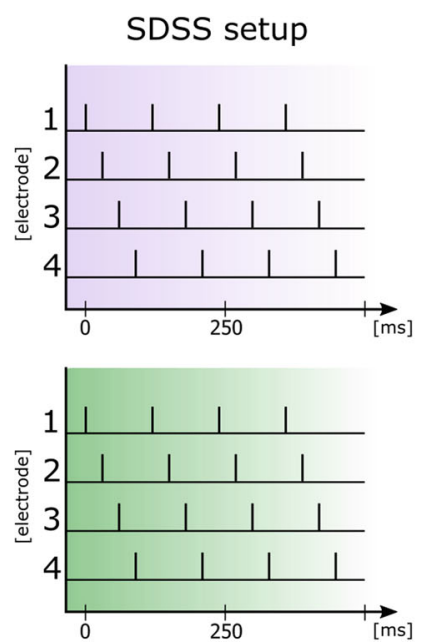
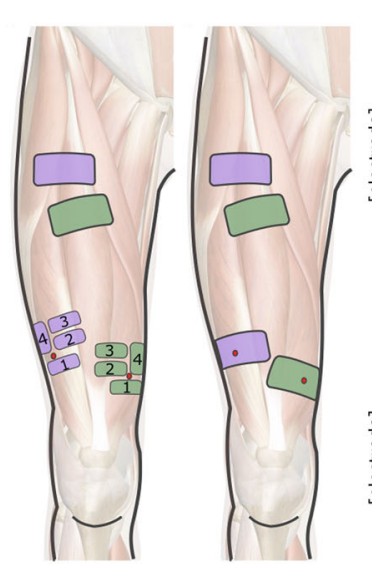

SES setup
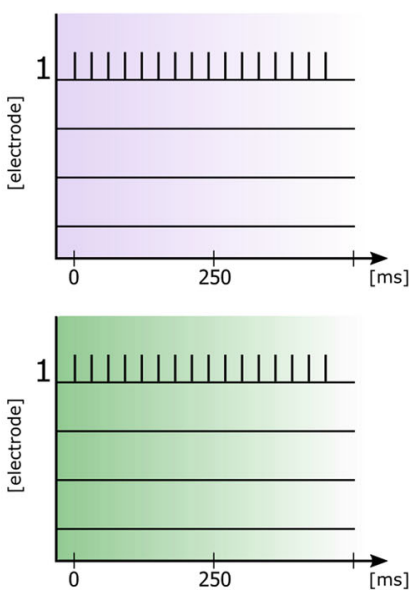

Fig. 1 Schematic picture of the electrode setup and the corresponding stimulation pattern. The purple and green electrodes are synchronised and stimulate synergistically the $\mathrm{m}$. lateralis (purple) and $\mathrm{m}$. medialis (green). Visible on the left: the SDSS setup with four small electrodes replacing the active electrodes. Electrodes were placed as close as possible to the located motor points. SES setup with two pairs of electrodes on the right side. Active electrodes were placed on the motor points of $\mathrm{m}$. vastus medialis and $\mathrm{m}$. vastus lateralis. Motor points are depicted with a red dot 
$\left(P_{\text {mean }}\right)$, peak power output $\left(P_{\text {peak }}\right)$ and the time from onset of the stimulation to $80 \%$ of $P_{\text {peak }}\left(t_{\text {peak } 80}\right)$ was calculated for each extension. For each of these three output parameters mean values and standard deviations were calculated for the initial 10 stimulated extensions (init), the final 20 extensions (final) and all 160 extenstions (overall) for each of the four subjects. $P_{\text {mean } L, R}$ is denoted as the mean power output of the left and right legs.

A fatigue index (FI) based on $P_{\text {mean }}$ describes the loss of power between the ten initial knee extensions $\left(P_{\text {init }}\right)$ and the final 20 knee extensions $\left(P_{\text {final }}\right)$ from the stimulated phase. Thus, $F I=1-\left(P_{\text {init }}-P_{\text {final }}\right) / P_{\text {init }}$. The higher the value, the higher the fatigue resistance; $F I=1$ means no fatigue.

\section{Results}

\section{Overall outcomes}

The SDSS setup gave higher $P_{\text {meanL, }, R}$ values for all four participants overall (increases of $132 \%$ in P1, 100\% in P2, $36 \%$ in P3 and $18 \%$ in P4 compared to SES, Fig. 2) and for the initial phase (increases of $84 \%, 59 \%, 66 \%$ and $16 \%$ ). Fatigue resistance was better with SDSS for P1, P2, P4 but worse for P3 ( 0.47 vs $0.35,0.63$ vs $0.49,0.90$ vs 0.82 and 0.59 vs 0.77 , respectively). No valid values were available for $P_{\text {peak } 80}$ and $t_{80}$ since not every stimulated extension had a clear peak.

The results are summarized in Table 2 and the individual power development of each leg is shown in Figs. 3, 4, 5 and 6.

\section{Individual cases}

\section{Case 1}

Participant P1 (data records in Fig. 3) had lesion level T3, AIS A, and markedly atrophied leg muscles. The measurements were conducted 24 months post injury

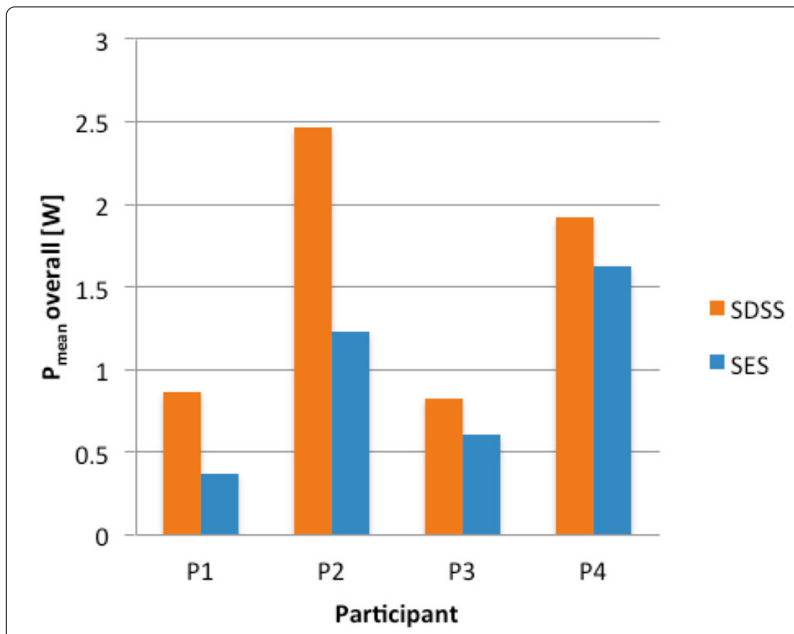

Fig. 2 Mean power response to all 160 extension measurements $\left(P_{\text {mean } L, R}\right.$ overall) for each participant at the second entry in the clinic (re-rehabilitation). This participant had muscle contractions in the abdominal region during the familiarization session (SES setup, right leg). Co-contractions of $\mathrm{m}$. sartorius and adductor muscles were visible during familiarization and small spastic leg activations were observed at the beginning of each measurement. The left leg showed only minor differences between the two stimulation setups, whereby the SDSS had a higher fatigue resistance (0.47 for SDSS vs. 0.35 for SES).

\section{Case 2}

Participant P2 (Fig. 4) had lesion level T6, AIS A, and the injury happened 5 months before the measurement, which took place in the last week of primary rehabilitation. $P_{\text {mean }}$ (overall) produced with the left leg was doubled for both setups compared to the output of the right leg (Table 2). This participant showed co-contractions in the hamstring muscles in all four measurements. The strongest co-contractions were observed during the first 2 min of the SDSS measurement in the right leg but they steadily decreased. Motor points were successfully detected in all stimulated muscles.

\section{Case 3}

Participant P3 (Fig. 5) had lesion level T2, AIS A, and the measurement took place 6 months after injury during primary rehabilitation. No co-contractions and very low muscle tone were observed. Motor points were only detected for the $\mathrm{m}$. vastus medialis on both legs. On both $\mathrm{m}$. vastus lateralis, the electrodes had to be placed based on guidelines from the literature and on experience.

\section{Case 4}

Participant P4 (Fig. 6) had lesion level T5, AIS A, and the measurement took place 6 months after injury during primary rehabilitation. Strong co-contractions in the hamstring muscles were observed in all four measurements, mainly at the beginning and then decreasing. This participant showed high muscle tone and the muscles twitched already when cold electrodes were being applied on the skin.

\section{Discussion}

The aim of this study was to compare the power output and fatigue properties of the quadricps femoris muscles in response to spatially distributed sequential stimulation (SDSS) versus traditional single electrode stimulation (SES) during a dynamic leg extension task in four untrained participants with motor-complete spinal cord injury. The task was designed to simulate knee joint movement in recumbent cycling for future applications with FES. 
Table 2 Power output, fatigue values and corresponding pulse width of each measurement

\begin{tabular}{|c|c|c|c|c|c|c|c|c|c|c|c|}
\hline \multirow[b]{3}{*}{ Participant } & \multirow[b]{3}{*}{ Leg } & \multicolumn{6}{|c|}{ Pmean [W] } & \multirow{2}{*}{\multicolumn{2}{|c|}{$\frac{\text { Fatigue }}{\text { Index }}$}} & \multicolumn{2}{|c|}{ Pulse width $[\mu \mathrm{s}]$} \\
\hline & & \multicolumn{2}{|c|}{ Initial } & \multicolumn{2}{|l|}{ Final } & \multicolumn{2}{|c|}{ Overall } & & & \multirow[b]{2}{*}{ SDSS } & \multirow[b]{2}{*}{ SES } \\
\hline & & $\overline{\text { SDSS }}$ & SES & SDSS & SES & SDSS & SES & SDSS & SES & & \\
\hline \multirow[t]{4}{*}{ P1 } & L & 1.54 & 1.53 & 0.74 & 0.47 & 0.93 & 0.69 & 0.48 & 0.31 & 176 & 196 \\
\hline & $\mathrm{R}$ & 1.37 & 0.05 & 0.61 & 0.08 & 0.80 & 0.05 & 0.45 & 1.69 & 188 & 128 \\
\hline & meanL,R & 1.45 & 0.79 & 0.68 & 0.27 & 0.86 & 0.37 & 0.47 & 0.35 & 182 & 162 \\
\hline & $\%$ diff & $84 \%$ & & $147 \%$ & & $132 \%$ & & $34 \%$ & & $12 \%$ & \\
\hline \multirow[t]{4}{*}{ P2 } & L & 4.92 & 3.18 & 2.66 & 1.60 & 3.37 & 1.86 & 0.54 & 0.50 & 185 & 195 \\
\hline & $\mathrm{R}$ & 1.75 & 1.01 & 1.52 & 0.44 & 1.56 & 0.60 & 0.87 & 0.44 & 180 & 192 \\
\hline & meanL, $R$ & 3.33 & 2.10 & 2.09 & 1.02 & 2.46 & 1.23 & 0.63 & 0.49 & 182.5 & 193.5 \\
\hline & $\%$ diff & $59 \%$ & & $104 \%$ & & $100 \%$ & & $28 \%$ & & $-5 \%$ & \\
\hline \multirow[t]{4}{*}{ P3 } & L & 1.87 & 1.14 & 0.94 & 0.77 & 1.14 & 0.90 & 0.50 & 0.67 & 240 & 250 \\
\hline & $\mathrm{R}$ & 0.55 & 0.31 & 0.48 & 0.34 & 0.51 & 0.32 & 0.88 & 1.11 & 250 & 240 \\
\hline & meanL,R & 1.21 & 0.73 & 0.71 & 0.56 & 0.83 & 0.61 & 0.59 & 0.77 & 245 & 245 \\
\hline & $\%$ diff & $66 \%$ & & $27 \%$ & & $36 \%$ & & $-23 \%$ & & $0 \%$ & \\
\hline \multirow[t]{4}{*}{ P4 } & L & 2.04 & 1.78 & 1.87 & 1.37 & 1.88 & 1.53 & 0.92 & 0.77 & 260 & 256 \\
\hline & R & 2.13 & 1.83 & 1.89 & 1.61 & 1.96 & 1.73 & 0.89 & 0.88 & 260 & 250 \\
\hline & meanL,R & 2.08 & 1.81 & 1.88 & 1.49 & 1.92 & 1.63 & 0.90 & 0.82 & 260 & 253 \\
\hline & $\%$ diff & $16 \%$ & & $26 \%$ & & $18 \%$ & & $10 \%$ & & $-3 \%$ & \\
\hline
\end{tabular}

\% diff: Percentage difference between SDSS > SES for parameter meanL, $R$

initial: first 10 extensions; final: last 20 extensions

In all four participants the mean power output during stimulated knee extension was higher with the SDSS setup. This outcome is in line with previous measurements with able-bodied participants with the same measurement protocol [44]. Three out of four participants showed higher fatigue resistance with the SDSS setup. However, the FI outcome is not as consistent as $P_{\text {mean }}$. These results suggests that SDSS is beneficial overall when compared to SES and that it may be beneficial in a wide range of FES applications. The following discussion analyses the differences between SDSS and SES in detail.

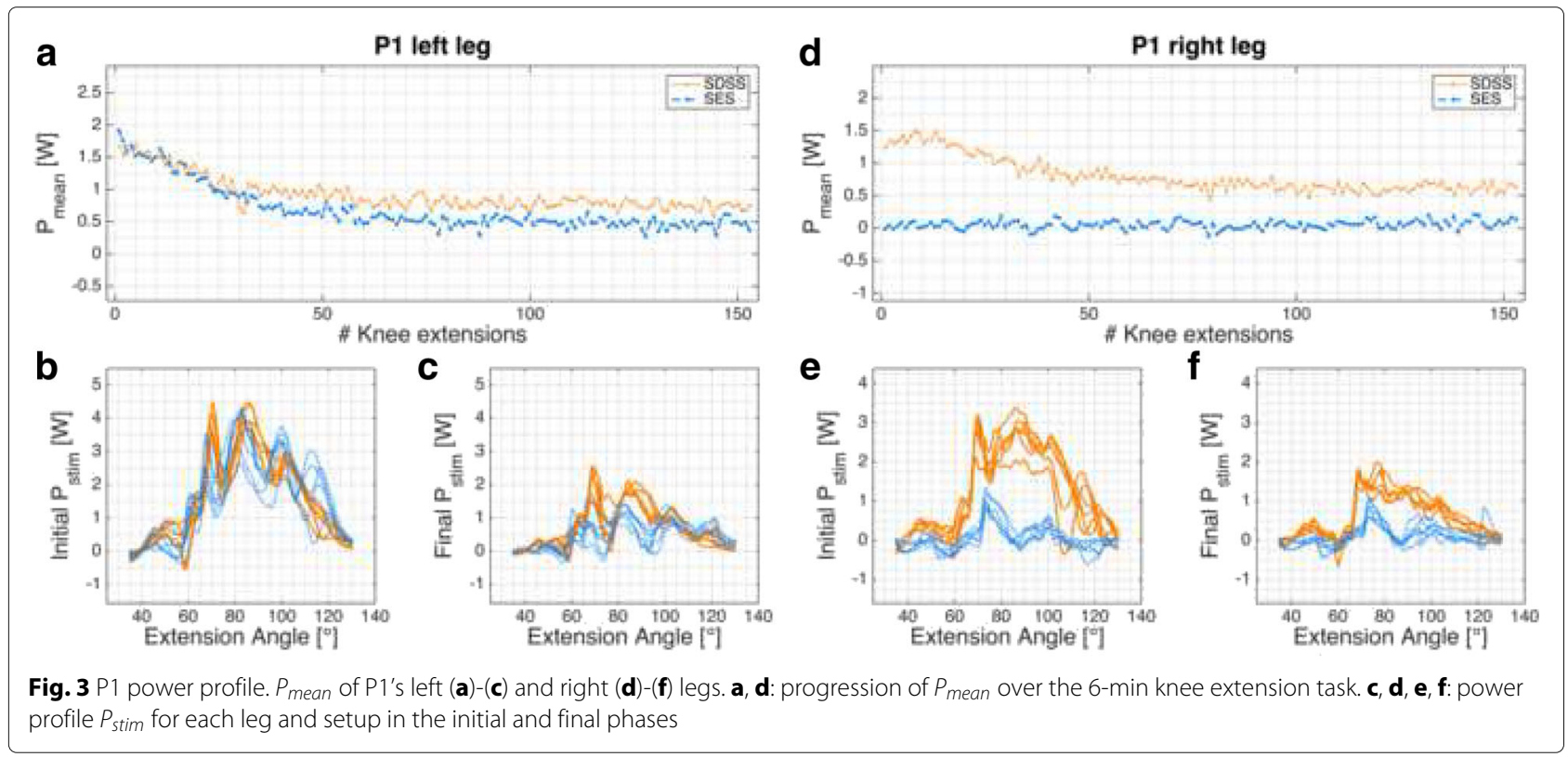



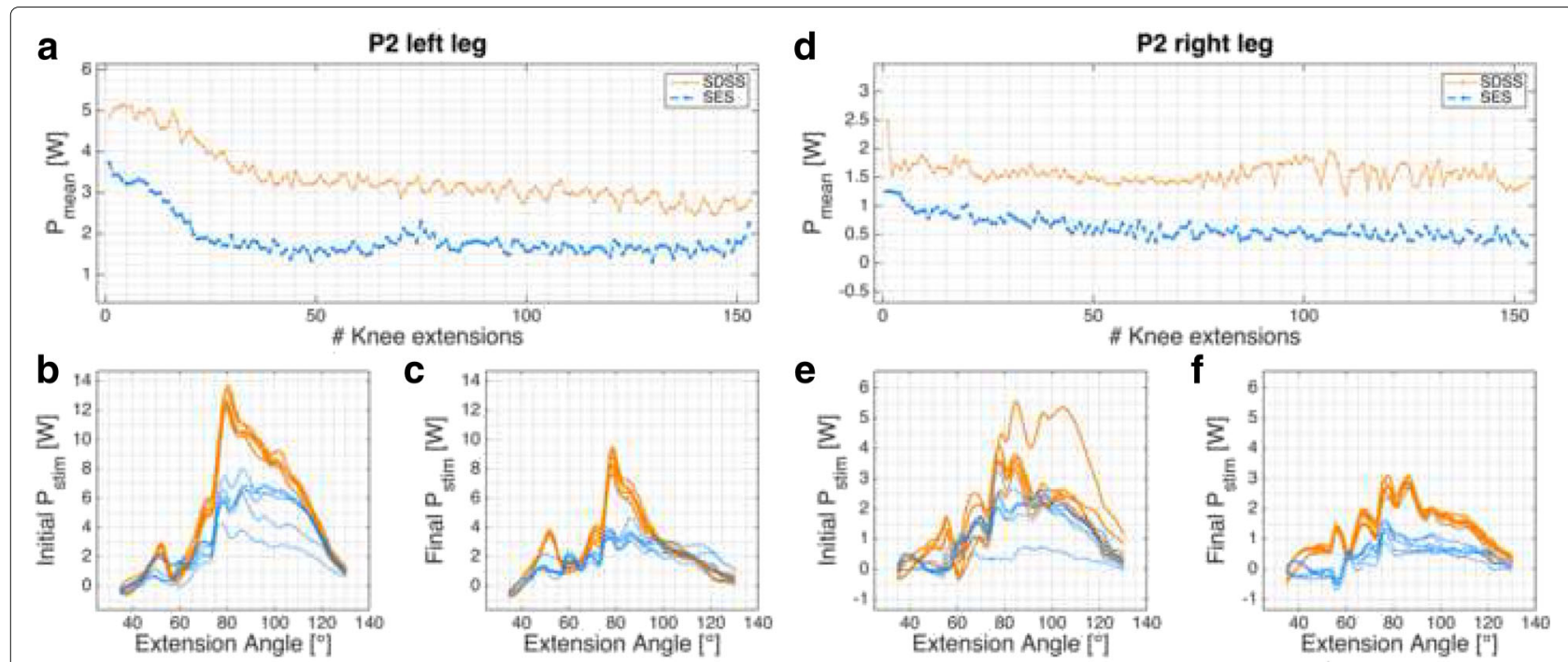

Fig. 4 P2 power profile. $P_{\text {mean }}$ of P2's left (a)-(c) and right (d)-(f) legs. a, d: progression of $P_{\text {mean }}$ over the 6-min knee extension task. c, d, e, f: power profile $P_{\text {stim }}$ for each leg and setup in the initial and final phases

\section{Power output}

Compared to previous measurements with able-bodied participants, the power output reached in this study was very low and likely not sufficient to perform functional tasks. All four participants were using FES for the first time and their muscles were untrained and already atrophied due to reduced muscle activity following the spinal cord injury. After such an injury, morphological and contractile changes occur in the muscles below the level of the lesion. A decrease in muscle cross-sectional area and reduced enzymatic activity leads to low muscle power as observed in participants P1 and P3 [50, 57, 58]. The measured power corresponds to the observed muscle tone and the time since injury. Participant P1, paralysed for almost two years, reported spastic reflexes, which might have preserved some muscle structure [30, 59]. Participant P3, in contrast, was paralysed for six months but showed very low muscle tone and motor points were only detected for vastus medialis on both legs. Another point often neglected in measuring spinal cord injured participants is the history before injury. Eser et al. [22] concluded that activity level before injury and thickness of the participant's fat layer is strongly related to

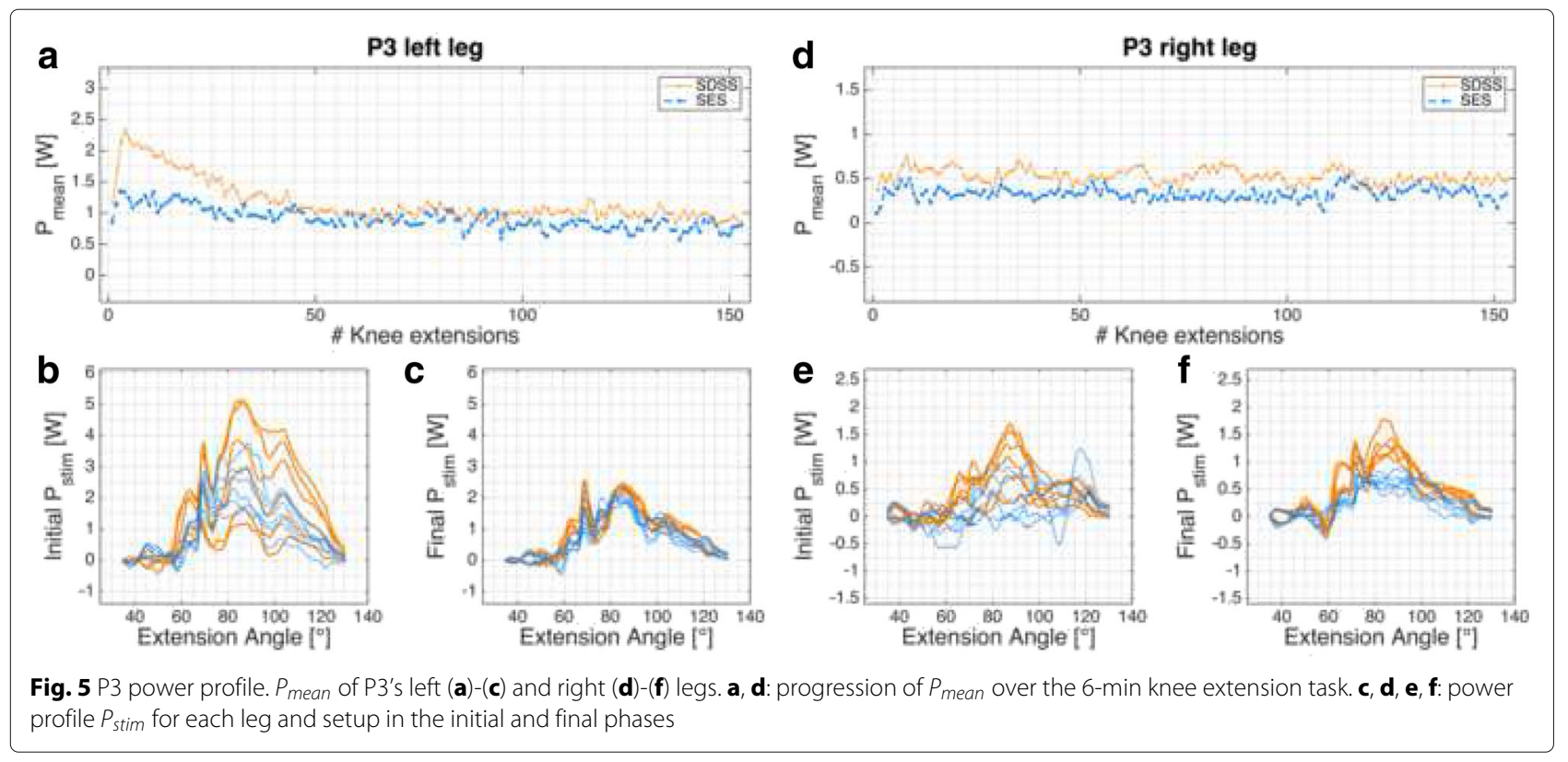



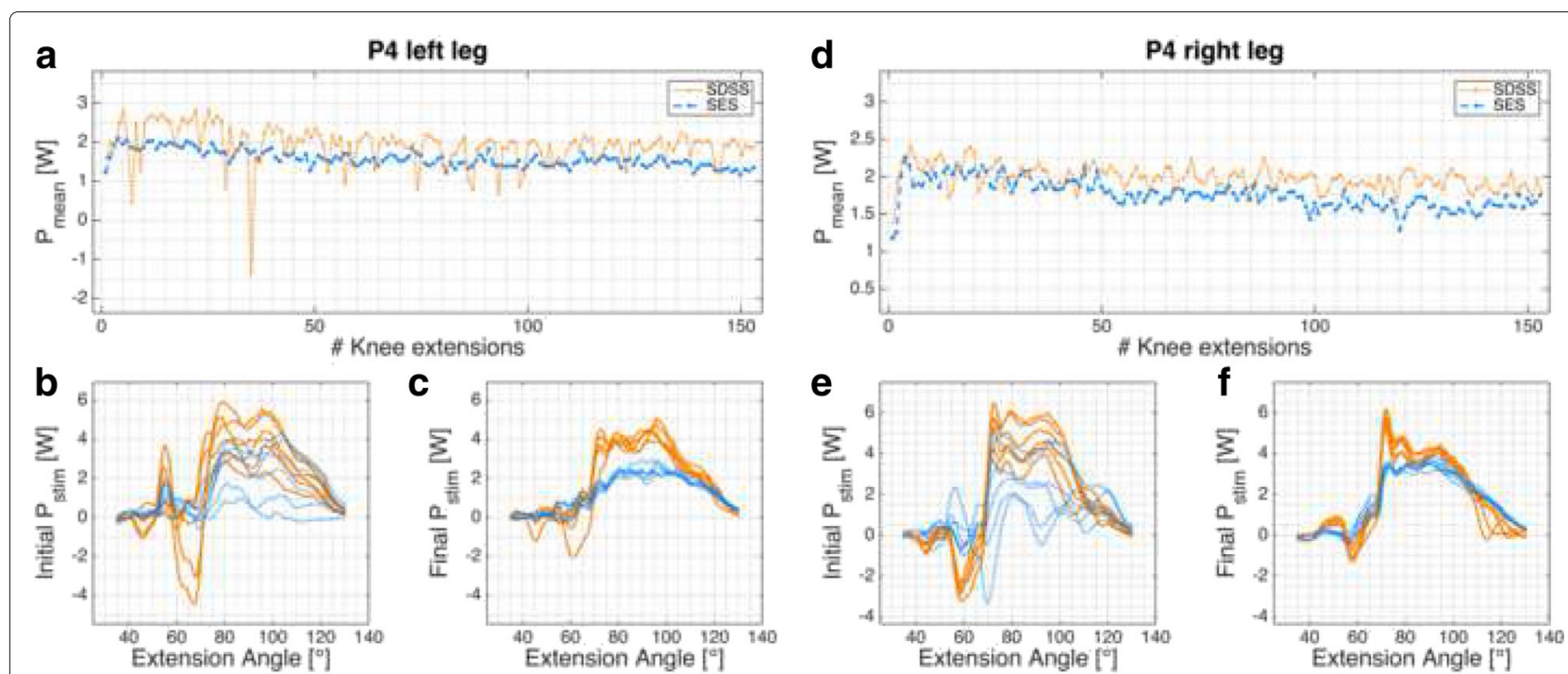

Fig. $6 \mathrm{P} 4$ power profile. $P_{\text {mean }}$ of P4's left (a)-(c) and right (d)-(f) legs. a, d: progression of $P_{\text {mean }}$ over the 6-min knee extension task. $\mathbf{c}, \mathbf{d}, \mathbf{e}, \mathbf{f}$ : power profile $P_{\text {stim }}$ for each leg and setup in the initial and final phases

the power output achieved by FES. Here, participants P2 and P4 were both aged between 20 and 30 years and reported being regularly physically active before injury. Participants P1 and P3 were aged between 40 and 50 years and reported no specific regular activities before injury. This might further explain the differences seen in power output. Nevertheless, in all four participants the electrical stimulation produced measurable muscle-based power responses. To regain strength and increase muscle cross-sectional area, repeated muscle stimulation over a longer period would be necessary [60-64].

Eight legs of four different participants were measured and in all cases the SDSS setup produced a higher power output compared to the conventional SES setup. With the exception of participant P1's left leg, the initial power output produced with SDSS was markedly superior to SES. Power is strongly related to the number of motor units recruited, so SDSS is probably able to recruit more motor units in SCI participants [65, 66]. Whether this increase in motor units comes from the larger surface area covered with SDSS, since the electrodes are more distributed than with SES (total electrode size is equal to SES) or through the deeper stimulation by the small electrodes remains unclear $[35,67,68]$. In all power profile plots SDSS produced sharper peaks (Figs. 3b, c, e, f, 4b, c, e, f, 5b, c, e, f, and $6 \mathrm{~b}, \mathrm{c}, \mathrm{e}, \mathrm{f}$ ). Especially in participant $\mathrm{P} 2$, the difference in power comes mainly from the sharp peak at $80 \mathrm{deg}$ knee angle (Fig. 4d). This may be due to more contracting fibres in deeper structures, which may be activated through higher current densities produced with SDSS electrodes $[69,70]$.

\section{Fatigue}

Stimulation intensity is strongly correlated with muscular fatigue, during both voluntary contractions and in artificial muscle stimulation $[15,71]$. In this study we increased pulse width during the familiarization process until the increase in force was no longer linearly related to the increased pulse width [63]. This had to be done very quickly, due to the possibility of fatigue onset, which would have influenced the subsequent measurement. This observation was done in real time using the screen of the Cybex device. Compared to the method used with ablebodied participants $[44,68]$, this method relies only on muscular properties and is not influenced by subjective pain tolerance, which is not applicable in persons with AIS-A SCI.

Three out of four participants showed increased fatigue resistance with SDSS (Table 2). Analysis of individual legs revealed that in two SES measurements final $P_{\text {mean }}$ was higher than initial $P_{\text {mean }}$ (Figs. $3 \mathrm{~d}, 5 \mathrm{~d}$ ). In both cases, the power output was very low and the difference of $0.03 \mathrm{~W}$ is marginal. Reducing joint and muscle resistance during the ongoing task is one possible explanation. Here, with SCI participants it is most probably due to reduced muscle tone resulting from FES [72]. On the other hand, both of these measurements gave a very low $P_{\text {mean }}$. Thus, the muscular load as well as the intensity were very low, which is consistent with previous observations that low intensity highly correlates with high fatigue resistance [21, 30, 73].

With regard to progression of knee extension (Figs. 3a, d, $4 a, d, 5 a, d$, and $6 a, d)$, in only six of 16 measurements the progression of the curve showed a rapid decline after $10-20$ knee extensions, as was seen in 
able-bodied participants [44]. Five of these six measurements were done with SDSS stimulation; only participant P1's left leg stimulated with SES showed the same shape of curve. All other measurements have shown a steady decline in $P_{\text {mean }}$ or fatigue index values higher than 0.8. Although a high fatigue index means good fatigue resistance, which is desirable, those high values strongly correlate with low $P_{\text {mean }}$ overall, which is suboptimal but in consistent to other reports in the literature $[15,19,21,71,73-75]$.

The steady decrease in force might be a sign of recruitment of slow-twitch fibres, whereas a rapid decrease of the initial $P_{\text {mean }}$ might indicate the activation of fast-twitch fibres. Participants P2, P3 and P4 were 6 months post injury and the measurements were conducted around the time at which oxidative enzymatic activity is expected to start to decline and the proportion of fibres that co-express both fast and slow myosin heavy chain isoforms increase [50, 76]. The composition of Type I and Type II fibres should not have changed substantially and might still be around $40 / 60$ for $\mathrm{m}$. lateralis and $\mathrm{m}$. rectus femoris [77]. Based on the review of Biering-Sorensen et al. [76], the measurement with participant P1 was 23 months post injury and therefore during a stable phase of the fibretype transformation process. However, implicating the recruited muscle fibre type based on these observations is not justified even though the type of muscle fibre recruited is clearly one parameter which influences the progression of the curve.

\section{Unintended stimulation}

In addition to changes in muscle fibre structure and loss of enzymatic activity, the whole body structure, such as fat mass and bone density, changes after a spinal cord injury [57]. The muscle's physiological properties influence the power and fatigue observed in response to electrical stimulation in a similar way to voluntary muscle contraction in able-bodied persons. In contrast to contractions which are voluntary or via implanted electrodes, muscle activation with surface electrodes has to overcome tissue resistance $[67,70,78]$. With SDSS the same amount of current is applied to a smaller surface area than during SES, which generates a higher current density and it is assumed that the resulting electrical field reaches deeper regions. In a functional task, this resistance is constantly changing due to muscle bulk shift and skin movement. Together with atrophied muscles and increased fat layers, it becomes more challenging to activate target muscles. It can happen that non-targeted nerves are stimulated as observed in participant P1's right leg. The stimulation was accompanied by uncomfortable contractions in the abdominal region despite the fact that the stimulation intensity was lower compared to the other measurements. Relocating the proximal electrodes during the familiarization did not help to reduce these contractions. Participants P2 and P4 had co-contractions with a short delay to the activated stimulation in all measurements in the hip flexor muscles. These muscles are activated through central pathways, which means the electrical signal was transmitted from sensory nerves via the spinal cord at levels L2/L3 to motor neurons. The co-contractions were strongest with SDSS and gradually decreasing [72]. In addition to hip flexor activity, participant $\mathrm{P} 4$ also had antagonistic muscle activity. Best visible as negative power at around $60 \mathrm{deg}$ and in the time plot of the left leg as negative outliers (Fig. 6a, b, e). The gradual decrease of these effects gave relatively high final $P_{\text {mean }}$ values, which, as noted above, explains the very high fatigue resistance in these measurements.

With regard to power output and fatigue properties, this study revealed some major benefits of the SDSS setup compared to SES in participants with motor- and sensorycomplete SCI. One limitation of the present study is the case-series design and the concomitant low number of participants. This does not mean that FES is not applicable for other persons with SCI with different impairment classification, but for homogeneity and to reduce confounding factors only persons with motor- and sensory-complete SCI were included. In a next step, the focus should be on recruiting more SCI participants with different grades of injury, and to recruit a sufficient number of participants to allow well-powered statistical analysis. The pilot data from the present study provide a basis for a sample-size and statistical-power calculation.

\section{Conclusions}

This study demonstrated higher power output for the SDSS setup in all eight leg-to-leg comparisons. Fatigue characteristics for the SDSS setup looks promising and SDSS seems to be more resistant when both setups activate the muscle at the same intensity level. Final $P_{\text {mean }}$ was higher for SDSS in all measurements. These outcomes are consistent with the results of previous measurements with able-bodied participants using the same test protocol [44] and demonstrate for the first time the promise of the SDSS approach in untrained people with SCI. Future studies should investigate long-term training protocols and compare SDSS and SES in SCI participants with well-trained muscles. Laubacher et al. [79] reported a single case which compared SDSS and SES in a well-trained person with $\mathrm{SCI}$ in the context of preparations for the FES bike race at Cybathlon 2016 in Zurich [80]. In this case, $P_{\text {meanL, } R}$ with SDSS was more than twice as high as $P_{\text {mean } L, R}$ with SES. These positive results should encourage the development of more sophisticated electrode setups for use in gross motor movements, such as in leg cycling. To date, several studies have shown increased power and fatigue resistance based on multi-electrode setups; combining these 
approaches with modulation of stimulation parameters might be a good future strategy to further increase power and fatigue resistance [81-83]. A further important aspect for better usability would be to apply multi-electrodes in cuffs or garments with an integrated method to determine the optimal stimulation setup.

\section{Acknowledgements}

The authors thank the following collaborators from the Swiss Paraplegic Centre, Nottwil: Dr Angela Frotzler and Dr Claudio Perret for their administrative support and Ines Bersch for her contribution to the protocol development.

\section{Funding}

This research was supported by the Swiss National Science Foundation (SNF-Nr:320030_15128/1)

\section{Availability of data and materials}

The datasets used and/or analysed during the current study are available from the corresponding author on reasonable request.

\section{Authors' contributions}

$\mathrm{ML}, \mathrm{SBM}, \mathrm{RR}$ and $\mathrm{KJH}$ defined the study protocol. $\mathrm{ML}$ and $\mathrm{AB}$ recruited the participants. ML and EAF developed the software for data recording and analysis. ML, EAF, AB performed the experiments. $M B$ was responsible for the medical check of the participants. ML wrote the manuscript and all authors revised the work critically, gave input and approved the final version.

\section{Ethics approval and consent to participate}

All procedures involving human participants were in accordance with the ethical standards of the institutional and/or national research committee and with the 1964 Helsinki declaration and its later amendments or comparable ethical standards. The local ethics committee (Ethics Committee northwest/central Switzerland, Ref.-Nr: BASEC 2016-00394) approved the study. All the patients gave their written informed consent to study participation.

\section{Consent for publication}

All the participants gave their written informed consent for publication of any individual person's anonymised data.

\section{Competing interests}

The authors declare that they have no competing interests.

\section{Publisher's Note}

Springer Nature remains neutral with regard to jurisdictional claims in published maps and institutional affiliations.

\section{Author details}

${ }^{1}$ Institute for Rehabilitation and Performance Technology, Division of Mechanical Engineering, Department of Engineering and Information Technology, Bern University of Applied Sciences, Pestalozzistrasse 20, 3400 Burgdorf, Switzerland. ${ }^{2}$ Sensory Motor Systems Lab, Department of Health Sciences and Technology, ETH Zurich, 8000 Zurich, Switzerland. ${ }^{3}$ Swiss Paraplegic Centre, 6207 Nottwil, Switzerland. ${ }^{4}$ Department of Physical Therapy, University of Delaware, Newark, United States of America.

Received: 6 August 2018 Accepted: 5 December 2018

Published online: 07 January 2019

\section{References}

1. Jacobs PL, Nash MS. Exercise recommendations for individuals with spinal cord injury. Sports Med. 2004;34(11):727-51.

2. Myers J, Lee M, Kiratli J. Cardiovascular disease in spinal cord injury: an overview of prevalence, risk, evaluation, and management. Am J Phys Med Rehabil. 2007:86(2):142-52.

3. Nash MS. Exercise as a health-promoting activity following spinal cord injury. J Neurol Phys Ther. 2005;29(2):87-103.

4. Bresnahan JJ, Farkas GJ, Clasey JL, Yates JW, Gater DR. Arm crank ergometry improves cardiovascular disease risk factors and community mobility independent of body composition in high motor complete spinal cord injury. J Spinal Cord Med. 2018;1-21. https://doi.org/10.1080/ 10790268.2017.1412562. https://www.ncbi.nlm.nih.gov/pubmed/ 29334345.

5. Creasey GH, Ho CH, Triolo RJ, Gater DR, DiMarco AF, Bogie KM, Keith MW. Clinical applications of electrical stimulation after spinal cord injury. J Spinal Cord Med. 2004;27(4):365-75.

6. Hagen EM. Acute complications of spinal cord injuries. World J Orthop. 2015;6(1):17-23.

7. Brurok B, Helgerud J, Karlsen T, Leivseth G, Hoff J. Effect of aerobic highintensity hybrid training on stroke volume and peak oxygen consumption in men with spinal cord injury. Am J Phys Med Rehabil. 2011;90(5):407-14

8. Davis GM, Hamzaid NA, Fornusek C. Cardiorespiratory, metabolic, and biomechanical responses during functional electrical stimulation leg exercise: health and fitness benefits. Artif Organs. 2008;32(8):625-9.

9. Decker MJ, Griffin L, Abraham LD, Brandt L. Alternating stimulation of synergistic muscles during functional electrical stimulation cycling improves endurance in persons with spinal cord injury. J Electromyogr Kinesiol. 2010;20(6):1163-9.

10. Dolbow DR, Gorgey AS, Gater DR, Moore JR. Body composition changes after 12 months of FES cycling: case report of a 60-year-old female with paraplegia. Spinal Cord. 2014;52 Suppl 1:3-4

11. Frotzler A, Coupaud S, Perret C, Kakebeeke TH, Hunt KJ, Donaldson NDN, Eser P. High-volume FES-cycling partially reverses bone loss in people with chronic spinal cord injury. Bone. 2008;43(1):169-76.

12. Bersch I, Tesini S, Bersch U, Frotzler A. Functional electrical stimulation in spinal cord injury: Clinical evidence versus daily practice. Artif Organs. 2015:39(10):849-54.

13. Popović DB. Advances in functional electrical stimulation (FES). J Electromyogr Kinesiol. 2014;24(6):795-802.

14. Duffell LD, de Donaldson NN, Newham DJ. Why is the metabolic efficiency of FES cycling low? IEEE Trans Neural Syst Rehabil Eng. 2009;17(3):263-9.

15. Gorgey AS, Black CD, Elder CP, Dudley GA. Effects of electrical stimulation parameters on fatigue in skeletal muscle. J Orthop Sports Phys Ther. 2009;39(9):684-92.

16. Hunt KJ, Fang J, Saengsuwan J, Grob M, Laubacher M. On the efficiency of FES cycling: A framework and systematic review. Technol Health Care. 2012;20(5):395-422.

17. Maffiuletti NA. Physiological and methodological considerations for the use of neuromuscular electrical stimulation. Eur J Appl Physiol. 2010;110(2):223-34

18. Newham DJ, Donaldson Nde N. FES cycling. Acta Neurochir Suppl. 2007;97(Pt 1):395-402.

19. Chou LW, Binder-Macleod S. The effects of stimulation frequency and fatigue on the force-intensity relationship for human skeletal muscle. Clin Neurophysiol. 2007;118(6):1387-96.

20. Deley G, Laroche D, Babault N. Effects of electrical stimulation pattern on quadriceps force production and fatique. Muscle Nerve. 2014;49(5):760-3.

21. Dreibati B, Lavet C, Pinti A, Poumarat G. Influence of electrical stimulation frequency on skeletal muscle force and fatigue. Ann Phys Rehabil Med. 2010;53(4):266-71.

22. Eser PC, Donaldson Nde N, Knecht H, Stussi E. Influence of different stimulation frequencies on power output and fatigue during FES-cycling in recently injured sci people. IEEE Trans Neural Syst Rehabil Eng. 2003;11(3):236-40.

23. Binder-Macleod S, Kesar T. Catchlike property of skeletal muscle: recent findings and clinical implications. Muscle Nerve. 2005;31(6):681-93.

24. Cometti C, Babault N, Deley G. Effects of constant and doublet frequency electrical stimulation patterns on force production of knee extensor muscles. PLOS ONE. 2016:11(5):0155429.

25. Scott WB, Lee SCK, Johnston TE, Binkley J, Binder-Macleod S. Effect of electrical stimulation pattern on the force responses of paralyzed human quadriceps muscles. Muscle Nerve. 2007;35(4):471-8.

26. Aksoz EA, Laubacher M, Binder-Macleod S, Hunt KJ. Effect of stochastic modulation of inter-pulse interval during stimulated isokinetic leg extension. Eur J Transl Myol. 2016;26(3):229-34.

27. Graham GM, Thrasher TA, Popovic MR. The effect of random modulation of functional electrical stimulation parameters on muscle fatigue. IEEE Trans Neural Syst Rehabil Eng. 2006;14(1):38-45. 
28. Graupe D, Suliga P, Prudian C, Kohn KH. Stochastically-modulated stimulation to slow down muscle fatigue at stimulated sites in paraplegics using functional electrical stimulation for leg extension. Neurol Res. 2000;22(7):703-4.

29. Thrasher A, Graham GM, Popovic MR. Reducing muscle fatigue due to functional electrical stimulation using random modulation of stimulation parameters. Artif Organs. 2005;29(6):453-8.

30. Gorgey AS, Dudley GA. The role of pulse duration and stimulation duration in maximizing the normalized torque during neuromuscular electrical stimulation. J Orthop Sports Phys Ther. 2008;38(8):508-16.

31. Gorgey AS, Mahoney E, Kendall T, Dudley GA. Effects of neuromuscular electrical stimulation parameters on specific tension. Eur J Appl Physiol. 2006;97(6):737-44.

32. Mesin L, Merlo E, Merletti R, Orizio C. Investigation of motor unit recruitment during stimulated contractions of tibialis anterior muscle. J Electromyogr Kinesiol. 2010;20(4):580-9.

33. Okuma Y, Bergquist AJ, Hong M, Chan KM, Collins DF. Electrical stimulation site influences the spatial distribution of motor units recruited in tibialis anterior. Clin Neurophysiol. 2013;124(11):2257-63.

34. Heckman CJ, Enoka RM. Motor unit. Compr Physiol. 2012;2(4):2629-82.

35. Reichel M, Breyer T, Mayr W, Rattay F. Simulation of the three-dimensional electrical field in the course of functional electrical stimulation. Artif Organs. 2002;26(3):252-5.

36. Bergquist AJ, Clair JM, Lagerquist O, Mang CS, Okuma Y, Collins DF. Neuromuscular electrical stimulation: implications of the electrically evoked sensory volley. Eur J Appl Physiol. 2011;111(10):2409-26.

37. Downey RJ, Bellman M, Kawai H, Gregory CM, Dixon W. Comparing the induced muscle fatigue between asynchronous and synchronous electrical stimulation in able-bodied and spinal cord injured populations. IEEE Trans Neural Syst Rehabil Eng. 2015;23(6):964-72.

38. Botter A, Oprandi G, Lanfranco F, Allasia S, Maffiuletti NA, Minetto MA. Atlas of the muscle motor points for the lower limb: implications for electrical stimulation procedures and electrode positioning. Eur J Appl Physiol. 2011;111(10):2461-71.

39. Gobbo M, Gaffurini P, Bissolotti L, Esposito F, Orizio C. Transcutaneous neuromuscular electrical stimulation: influence of electrode positioning and stimulus amplitude settings on muscle response. Eur J Appl Physiol. 2011;111(10):2451-9.

40. Gobbo M, Maffiuletti NA, Orizio C, Minetto MA. Muscle motor point identification is essential for optimizing neuromuscular electrical stimulation use. J Neuroeng Rehabil. 2014;11:17.

41. Pournezam M, Andrews BJ, Baxendale RH, Phillips GF, Paul JP. Reduction of muscle fatigue in man by cyclical stimulation. J Biomed Eng. 1988;10(2):196-200.

42. Popovic LZ, Malesevic NM. Muscle fatigue of quadriceps in paraplegics: comparison between single vs. multi-pad electrode surface stimulation. Conf Proc IEEE Eng Med Biol Soc. 2009;2009:6785-8.

43. Malesevic NM, Popovic LZ, Schwirtlich L, Popovic DB. Distributed low-frequency functional electrical stimulation delays muscle fatigue compared to conventional stimulation. Muscle Nerve. 2010;42(4):556-62.

44. Laubacher M, Aksoz AE, Riener R, Binder-Macleod S, Hunt KJ. Power output and fatigue properties using spatially distributed sequential stimulation in a dynamic knee extension task. Eur J Appl Physiol. 2017;117(9):1787-98

45. Bergquist AJ, Babbar V, Ali S, Popovic MR, Masani K. Fatigue reduction during aggregated and distributed sequential stimulation. Muscle Nerve. 2017;56(2):271-81.

46. Sayenko DG, Nguyen R, Popovic MR, Masani K. Reducing muscle fatigue during transcutaneous neuromuscular electrical stimulation by spatially and sequentially distributing electrical stimulation sources. Eur J Appl Physiol. 2014;114(4):793-804.

47. Nguyen R, Masani K, Micera S, Morari M, Popovic MR. Spatially distributed sequential stimulation reduces fatigue in paralyzed triceps surae muscles: a case study. Artif Organs. 2011;35(12):1174-80.

48. Broman H, De Luca CJ, Mambrito B. Motor unit recruitment and firing rates interaction in the control of human muscles. Brain Res. 1985;337(2): 311-9.

49. De Luca CJ, LeFever RS, MCCue MP, Xenakis AP. Behaviour of human motor units in different muscles during linearly varying contractions. J Physiol. 1982;329:113-28.

50. Castro MJ, Apple Jr DF, Hillegass EA, Dudley GA. Influence of complete spinal cord injury on skeletal muscle cross-sectional area within the first 6 months of injury. Eur J Appl Physiol Occup Physiol. 1999;80(4):373-8.
51. Dudley-Javoroski S, Shields RK. Muscle and bone plasticity after spinal cord injury: review of adaptations to disuse and to electrical muscle stimulation. J Rehabil Res Dev. 2008;45(2):283-96.

52. Malisoux L, Jamart C, Delplace K, Nielens H, Francaux M, Theisen D. Effect of long-term muscle paralysis on human single fiber mechanics. J Appl Physiol (1985). 2007;102(1):340-9.

53. Livshitz LM, Mizrahi J, Einziger PD. Interaction of array of finite electrodes with layered biological tissue: effect of electrode size and configuration. IEEE Trans Neural Syst Rehabil Eng. 2001;9(4):355-61.

54. Huang $Y$, Zhen Z, Song Y, Zhu Q, Wang S, Liu J. Motor training increases the stability of activation patterns in the primary motor cortex. PLOS ONE. 2013;8(1):53555.

55. Martin R, Sadowsky C, Obst K, Meyer B, McDonald J. Functional electrical stimulation in spinal cord injury:: from theory to practice. Top Spinal Cord Inj Rehabil. 2012;18(1):28-33.

56. Carey TS, Boden SD. A critical guide to case series reports. Spine (Phila Pa 1976). 2003;28(15):1631-4.

57. Giangregorio L, McCartney N. Bone loss and muscle atrophy in spinal cord injury: epidemiology, fracture prediction, and rehabilitation strategies. J Spinal Cord Med. 2006;29(5):489-500.

58. Vandenborne K, Elliott MA, Walter GA, Abdus S, Okereke E, Shaffer M, Tahernia D, Esterhai JL. Longitudinal study of skeletal muscle adaptations during immobilization and rehabilitation. Muscle Nerve. 1998;21(8): 1006-12.

59. Ditor DS, Hamilton S, Tarnopolsky MA, Green HJ, Craven BC, Parise G, Hicks AL. Na+,k+-atpase concentration and fiber type distribution after spinal cord injury. Muscle Nerve. 2004;29(1):38-45.

60. Adams GR, Harris RT, Woodard D, Dudley GA. Mapping of electrical muscle stimulation using mri. J Appl Physiol. 1993;74(2):532-7.

61. Berry HR, Kakebeeke TH, Donaldson N, Perret C, Hunt KJ. Energetics of paraplegic cycling: adaptations to 12 months of high volume training. Technol Health Care. 2012;20(2):73-84.

62. Chilibeck PD, Jeon J, Weiss C, Bell G, Burnham R. Histochemical changes in muscle of individuals with spinal cord injury following functional electrical stimulated exercise training. Spinal Cord. 1999;37(4):264-8.

63. Duffell LD, Donaldson N. d. N., Perkins TA, Rushton DN, Hunt KJ Kakebeeke TH, Newham DJ. Long-term intensive electrically stimulated cycling by spinal cord-injured people: effect on muscle properties and their relation to power output. Muscle Nerve. 2008;38(4):1304-11.

64. Gorgey AS, Khalil RE, Lester RM, Dudley GA, Gater DR. Paradigms of lower extremity electrical stimulation training after spinal cord injury. J Vis Exp. 2018;132:e57000.

65. Hodson-Tole EF, Wakeling JM. Motor unit recruitment for dynamic tasks: current understanding and future directions. J Comp Physiol B. 2009;179(1):57-66.

66. Rubinstein $\mathrm{S}$, Kamen $\mathrm{G}$. Decreases in motor unit firing rate during sustained maximal-effort contractions in young and older adults. J Electromyogr Kinesiol. 2005;15(6):536-43.

67. Kuhn A, Keller T, Lawrence M, Morari M. The influence of electrode size on selectivity and comfort in transcutaneous electrical stimulation of the forearm. IEEE Trans Neural Syst Rehabil Eng. 2010;18(3):255-62.

68. Laubacher M, Aksoz EA, Binder-Macleod S, Hunt KJ. Comparison of proximally versus distally placed spatially distributed sequential stimulation electrodes in a dynamic knee extension task. Eur J Transl Myol. 2016;26(2):110-5.

69. Alon G, Kantor G, Ho HS. Effects of electrode size on basic excitatory responses and on selected stimulus parameters. J Orthop Sports Phys Ther. 1994;20(1):29-35.

70. Keller T, Kuhn A. Electrodes for transcutaneous (surface) electrical stimulation. J Autom Control. 2008;18(2):35-45.

71. Binder-Macleod S, Halden EE, Jungles KA. Effects of stimulation intensity on the physiological responses of human motor units. Med Sci Sports Exerc. 1995;27(4):556-65.

72. Elbasiouny SM, Moroz D, Bakr MM, Mushahwar VK. Management of spasticity after spinal cord injury: current techniques and future directions. Neurorehabil Neural Repair. 2010;24(1):23-33.

73. Kesar T, Chou L-W, Binder-Macleod S. Effects of stimulation frequency versus pulse duration modulation on muscle fatigue. J Electromyogr Kinesiol. 2008;18(4):662-71.

74. Binder-Macleod S, Guerin T. Preservation of force output through progressive reduction of stimulation frequency in human quadriceps femoris muscle. Phys Ther. 1990;70(10):619-25. 
75. Gregory CM, Dixon W, Bickel CS. Impact of varying pulse frequency and duration on muscle torque production and fatigue. Muscle Nerve. 2007;35(4):504-9.

76. Biering-Sorensen B, Kristensen IB, Kjaer M, Biering-Sorensen F. Muscle after spinal cord injury. Muscle Nerve. 2009;40(4):499-519.

77. Saltin B, Henriksson J, Nygaard E, Andersen P, Jansson E. Fiber types and metabolic potentials of skeletal muscles in sedentary man and endurance runners. Ann N Y Acad Sci. 1977;301:3-29.

78. Livshitz LM, Einziger PD, Mizrahi J. Current distribution in skeletal muscle activated by functional electrical stimulation: image-series formulation and isometric recruitment curve. Ann Biomed Eng. 2000;28:1218-28.

79. Laubacher M, Aksoz EA, Bersch I, Hunt KJ. The road to Cybathlon 2016 functional electrical stimulation cycling team IRPT/SPZ. Eur J Transl Myol. 2017;27(4):259-64.

80. Riener R. The cybathlon promotes the development of assistive technology for people with physical disabilities. J NeuroEngineering Rehabil. 2016;13(1):49.

81. Brown TI, Huang Y, Morgan DL, Proske U, Wise A. A new strategy for controlling the level of activation in artificially stimulated muscle. IEEE Trans Rehabil Eng. 1999;7(2):167-73.

82. Doucet BM, Lam A, Griffin L. Neuromuscular electrical stimulation for skeletal muscle function. Yale J Biol Med. 2012;85(2):201-15.

83. Downey RJ, Bellman M, Sharma N, Wang Q, Gregory CM, Dixon W. A novel modulation strategy to increase stimulation duration in neuromuscular electrical stimulation. Muscle Nerve. 2011;44(3):382-7.

Ready to submit your research? Choose BMC and benefit from:

- fast, convenient online submission

- thorough peer review by experienced researchers in your field

- rapid publication on acceptance

- support for research data, including large and complex data types

- gold Open Access which fosters wider collaboration and increased citations

- maximum visibility for your research: over 100M website views per year

At $B M C$, research is always in progress.

Learn more biomedcentral.com/submission 